\title{
Arte e salute
}

\section{Sergio Marengo}

Il congresso 2016 dell'unione mondiale scrittori medici si è svolto a Garlate (Lecco, Italia) dal 14 al 18 settembre, sotto il patrocinio dell'UMEM. Il lago di Como, a Lecco, ha un particolare fascino letterario, poiché in questi luoghi è stato ambientato il libro I promessi sposi di Alessandro Manzoni, massimo romanziere italiano.

\section{Essere medici e scrittori non è un'eccezione.}

La partecipazione al congresso è stata notevole e l'organizzazione dell'AMSI (Ass. Medici Scrittori Italiani) impeccabile. Diverse le rappresentanze nazionali, tra cui Italia, Bulgaria, Ecuador, Francia, Germania, Polonia, Portogallo, Romania, Spagna e Svizzera. Molti testi avevano traduzioni in più lingue. Il tema principale era "Arte e Salute». Essere medici e scrittori non è un'eccezione. Ciò è confermato da vari esempi, tra cui Anton Čechov, Georges Duhamel, Arthur Conan

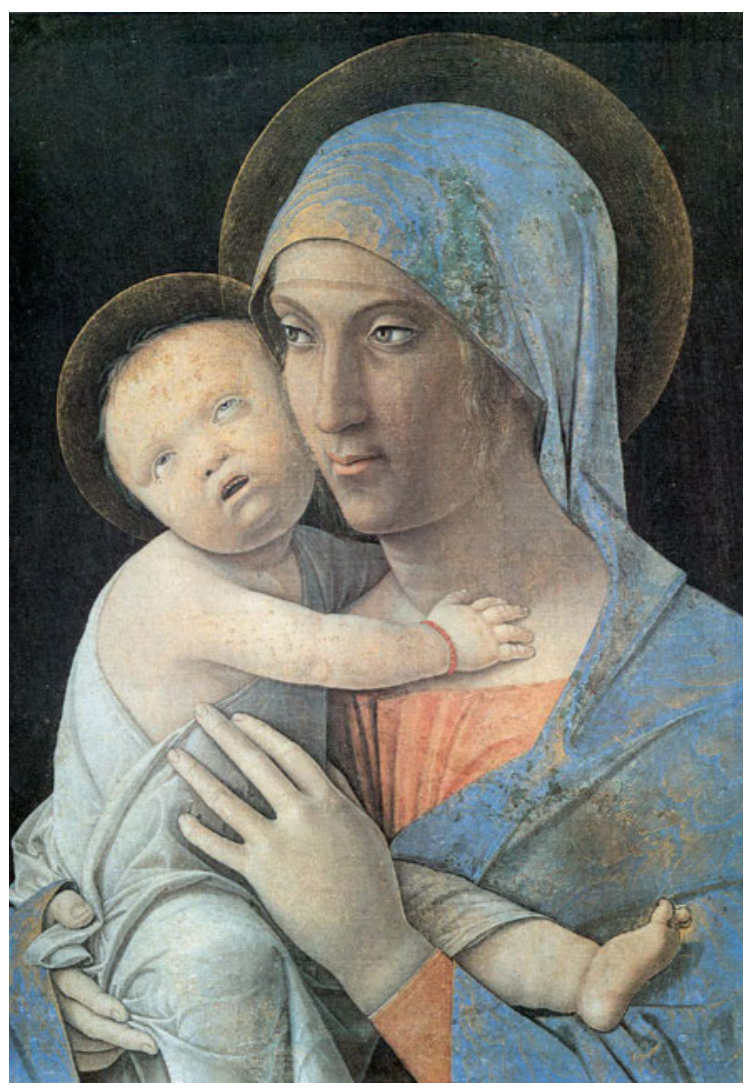

Dipinto Madonna col Bambino di Andrea Mantegna. Provenienza: Accademia Carrara, Bergamo, Italia.
Doyle, Jean-Christophe Rufin e Laurent Seksik. La Dottoressa Sylvie Claire Briand ha discusso sui rapporti tra letteratura ed epidemie ad alta letalità; queste pongono in pericolo il legame sociale e la coesione delle comunità. Gianfranco Brini ha esposto su Anton Čechov, medico e grande letterato.

\section{L'arte puo alleggerire i dolori}

Roland Noël, presidente dei medici scrittori francesi, ha parlato di Paul Verlaine, il sensibile, di Renoir, il coraggioso, affetto da reumatismo poliarticolare de-

\section{Il famoso dipinto Le Bagnanti fu terminato in condizioni fisiche "terribili».}

formante. Per quest'ultimo, solo l'arte poteva alleviare i dolori. Il famoso dipinto Le Bagnanti fu terminato in condizioni fisiche «terribili». Claude Ploceniak ha esposto il tema «Il magnesio e la storia». La carenza di magnesio è nota da molto tempo, ma in passato non era stata semplicemente diagnosticata. Helga Thomas, in una poesia, rileva l'affinità tra l'acqua e l'amore: non possono vivere l'una senza l'altro. La presidente dei medici scrittori italiani, Patrizia Valpiani, ha illustrato il periodo cechoviano, la sua poetica e due poesie (L'odore dell'inverno e L'esprit de Paris). Abraham de Voogt è intervenuto sul tema «Mondo disumanizzato, senza amore». L'esperienza prova che l'aggressività degli animali è parallela alla loro concentrazione mentale; così è anche per l'uomo. Ufficiale rappresentante dell'UMEM è stata la sua segretaria generale, Françoise Verrey Bass di Bienne, pure presidente dell'ASEM, l'associazione svizzera degli scrittori medici. Un ricordo, difficile da dimenticare, è stato la visita alla Pinacoteca Carrara di Bergamo, i cui quadri ci hanno fatto sognare. Anche la serata conclusiva si è svolta in un'atmosfera d'amicizia e di generale apprezzamento.

Termino riportando le conclusioni di Suzanne Rafflé, chirurgo dentista: «Grâce aux nourritures des œuvres de l'esprit, nous aurons dans notre panthéon des souvenirs, mais pas de regrets.»

\section{Credito fotografico}

Wikipedia: https://it.wikipedia.org/wiki/Madonna_col_

Bambino_\%28Mantegna_Bergamo\%29\#/media/File:Mantegna,_madonna_dell\%27accademia_carrara_di_bergamo.jpg 